
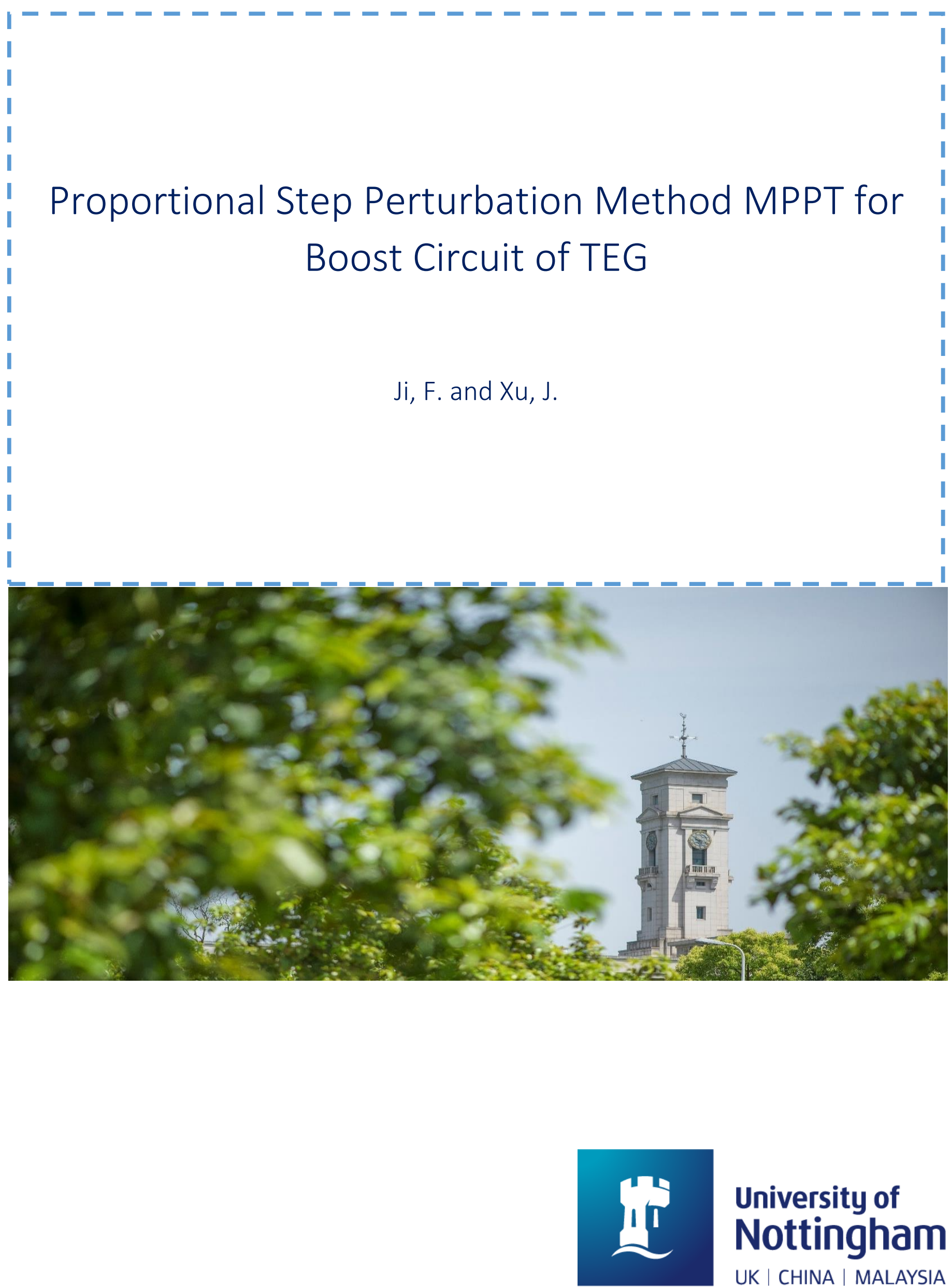
University of Nottingham Ningbo China, 199 Taikang East Road, Ningbo, 315100, Zhejiang, China.

First published 2020

This work is made available under the terms of the Creative Commons Attribution 4.0 International License:

http://creativecommons.org/licenses/by/4.0

The work is licenced to the University of Nottingham Ningbo China under the Global University Publication Licence:

https://www.nottingham.edu.cn/en/library/documents/researchsupport/global-university-publications-licence-2.0.pdf 


\title{
Proportional Step Perturbation Method MPPT for Boost Circuit of TEG
}

\author{
Feng Ji ${ }^{(凹)} 1$ and John $\mathrm{Xu}^{2}$ \\ University of Nottingham Ningbo China
}

\begin{abstract}
In order to improve the working efficiency of thermoelectric generation (TEG), maximum power point tracking (MPPT) is usually used in the system. The perturbation method is a traditional method for MPPT. The perturbation of the step length is normally fixed. It will lead to be not accurate enough if the step size is too big. If the step size is too small, it will lead to take a long time. Therefore, the proportional step perturbation method MPPT is proposed in the paper. This method can be implemented by using large steps at the beginning to save time. When it is near the maximum power point, small steps can be used to achieve the accurate purpose. The derivation of the method has been described in details in the paper. The simulation model is established based on Matlab. The simulation results show that the method is feasible.
\end{abstract}

Keywords: Boost, MPPT, Proportional Step Perturbation Method

\section{$1 \quad$ Introduction}

At present, the MPPT methods include fixed voltage method, disturbance observation method, conductance increment method, etc. The constant voltage tracking method is a kind of approximate maximum power tracking method. The constant voltage tracking method has certain power loss. When the temperature changes, the open circuit voltage of the thermoelectric chip will change accordingly. Therefore, the tracking efficiency is not high enough. And the MPPT fixed point is not accurate enough[1-3]. The incremental conductance method is accurate in control and quick in response. It is suitable for the situation where atmospheric conditions change rapidly. However, the hardware requirements are high such as the electronic sensor accuracy. And the response speed of each part of the system is required to be fast. Therefore, the hardware cost of the whole system is also relatively high. When the accuracy of the sensor is limited, there will be errors in the calculation of the increment and instantaneous conductance of the thermoelectric module by the processor. It will inevitably lead to inaccurate tracking[4-6]. For the traditional perturbation method with fixed step length, the output of the thermoelectric panel will be floating around maximum power point if $\Delta \mathrm{U}$ value is too large. If $\Delta \mathrm{U}$ value is small, it can guarantee the tracking accuracy. But this will need more time. When the maximum power point change frequently, the result will be worse[7-9]. Therefore, the proportional step perturbation method MPPT is proposed in this paper. It can overcome the shortcomings of the traditional method and ensure fast and accurate MPPT. 


\section{Boost Circuit with PID Controller}

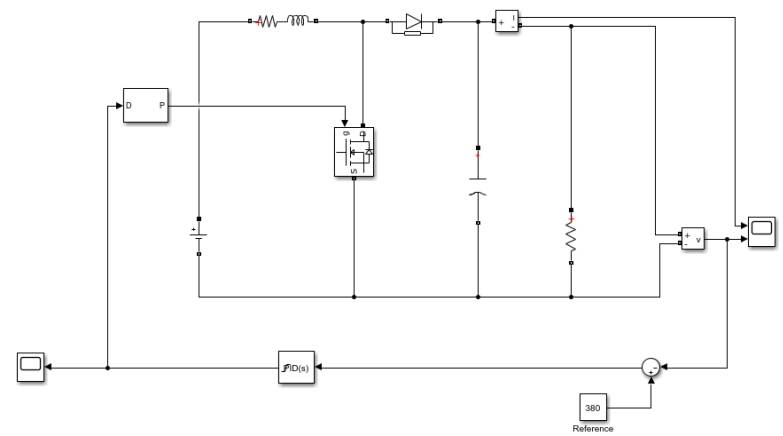

Fig. 1. Boost Circuit

When the IGBT conducts in the circuit, the current forms a circuit by $E_{0}$ through the boost inductance L. And the inductance L stores energy. When the IGBT is turned off, the reverse electromotive force generated by the inductance and the DC power supply are superimposed on the working load. Therefore, it can obtain a higher voltage on the load. The function of the diode is to ensure a single direction pass. And the current $i_{L}$ continues to flow through the diode D. By adjusting the on-off period of the switch device IGBT, the output current and voltage on the load side can be adjusted.

$$
U_{0}=\frac{t_{o n}+t_{o f f}}{t_{o f f}} E_{0}
$$

In the above equation (1), $\mathrm{T}$ is the switching period, $t_{\text {on }}$ is the conduction time and $t_{o f f}$ is the turn-off time. The circuit adopts closed-loop voltage control as shown in the Fig. 2. It is the schematic diagram of the closed-loop voltage control system. The voltage loop is controlled by the constant value. The voltage controller adopts PID controller. Therefore, the output voltage can obtain accurate and stable value.

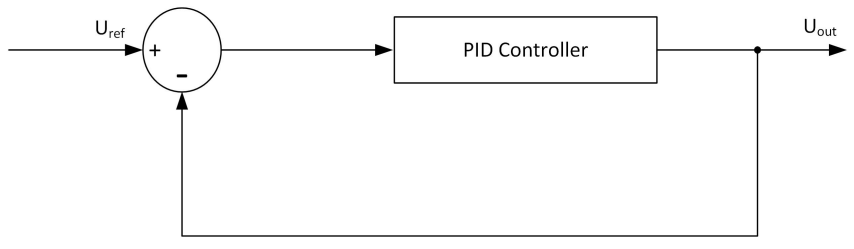

Fig. 2. Voltage PID Control Mode 
Fig.3 is the output under normal conditions for the Boost circuit. And Fig.4 is the output under sudden load change for the Boost circuit. The waveform shows that it can obtain stable output under different working load.
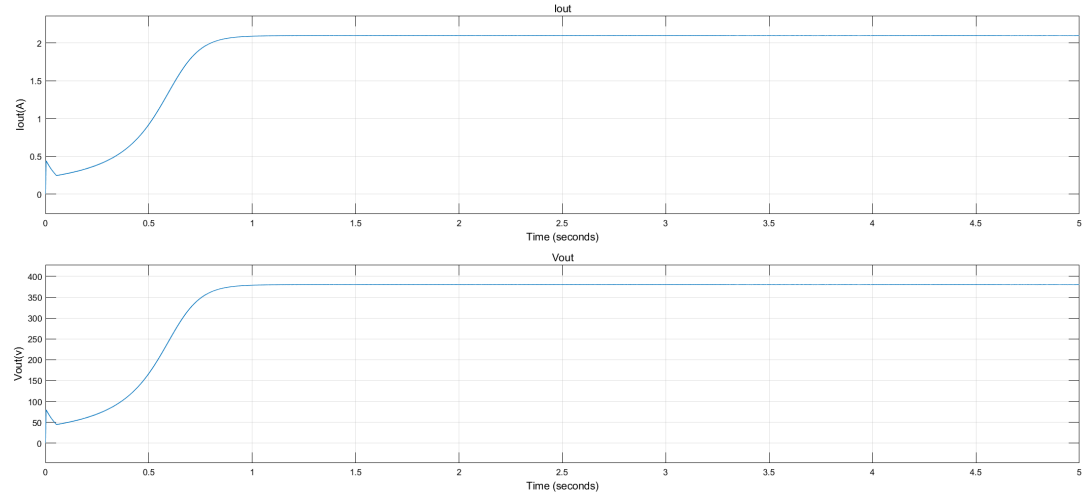

Fig. 3. Output Under Normal Conditions of Boost Circuit
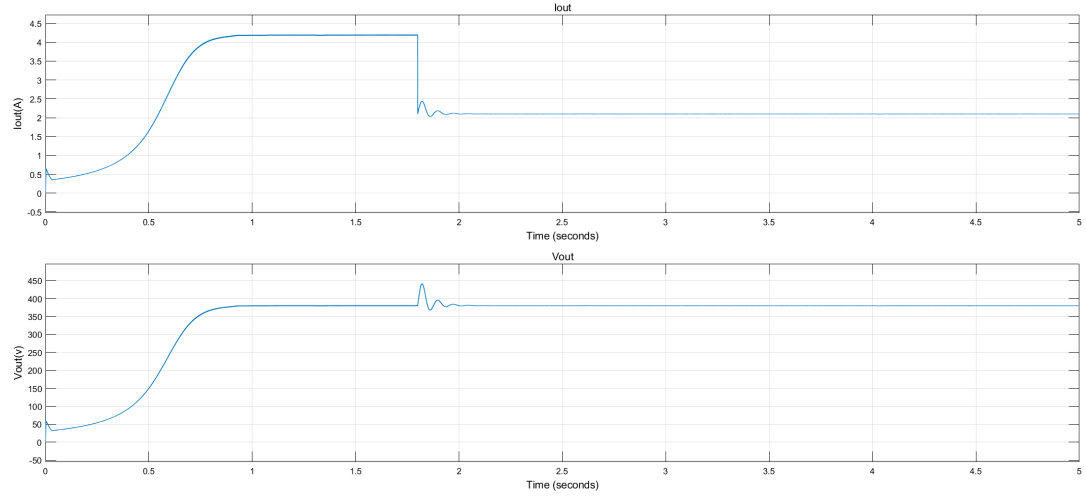

Fig. 4. Output Under Sudden Load Change of Boost Circuit

\section{Proportional Step Perturbation MPPT}

Because of the slow temperature changing speed, it can be considered that the temperature at both ends of the thermoelectric generator is constant in a control cycle. The circuit model can be equivalent to a voltage source and a fixed resistance in series as shown in Fig.5. 
Feng Ji et al.

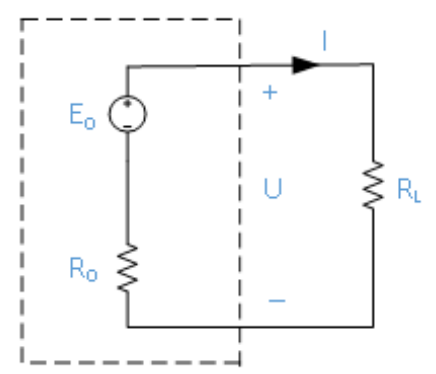

Fig. 5. Thermoelectric Circuit Model

Fig.5 is a simple closed circuit, $R_{L}$ is the load resistance of the external circuit. The terminal voltage is:

$$
U_{0}=E_{0}-R E_{0}
$$

Multiply the current I on both sides of the equation:

$$
I U=I E_{0}-I^{2} R_{0}
$$

Assuming the load is pure resistance, $\mathrm{I}^{*} E_{0}$ is the total power of the circuit, $I^{2} R_{0}$ is the power of internal resistance and $\mathrm{I}^{*} \mathrm{U}$ is the power of output.

$$
P=I U=I^{2} R L=R_{L}\left[\frac{E_{0}}{\left(R_{L}+R_{0}\right)}\right]^{2}=\frac{R_{L} E_{0}^{2}}{\left(R_{L}+R_{0}\right)^{2}}
$$

Because of:

$$
\left(R_{L}+R_{0}\right)^{2}=\left(R_{L}-R_{0}\right)^{2}+4 R_{L} R_{0}
$$

So:

$$
P=\frac{R_{L} E_{0}^{2}}{\left(R_{L}+R_{0}\right)^{2}}=\frac{R_{L} E_{0}^{2}}{\left(R_{L}-R_{0}\right)^{2}+4 R_{L} R_{0}}=\frac{E_{0}^{2}}{\frac{\left(R_{L}-R_{0}\right)^{2}}{R_{L}}+4 R_{0}}
$$

Since the EMF $E_{0}$ and internal resistance $R_{0}$ of the circuit are independent of the external circuit, they can be regarded as constant. When the load resistance $R_{L}$ is equal to the internal resistance $R_{0}$, the output power of the circuit reaches the maximum value. Its maximum value is:

$$
P_{m}=\frac{E_{0}^{2}}{4 R_{0}}=\frac{E_{0}^{2}}{4 R_{L}}
$$

The short-circuit current is IS. When $R_{0}=R_{L}$, the equivalent circuit of the thermoelectric generation is working at the maximum power point (MPP).Then the following two conditions are also met with. 


$$
\begin{gathered}
U=\frac{E_{0}}{2} \\
I_{s}=\frac{E_{0}}{R_{0}}=\frac{2 E_{0}}{R_{0}+R_{L}}=2 I
\end{gathered}
$$

The thermoelectric generator is connected to DC / DC boost circuit as shown in the Fig.6. There are two modes in normal operation: MOSFET on mode and MOSFET off mode.

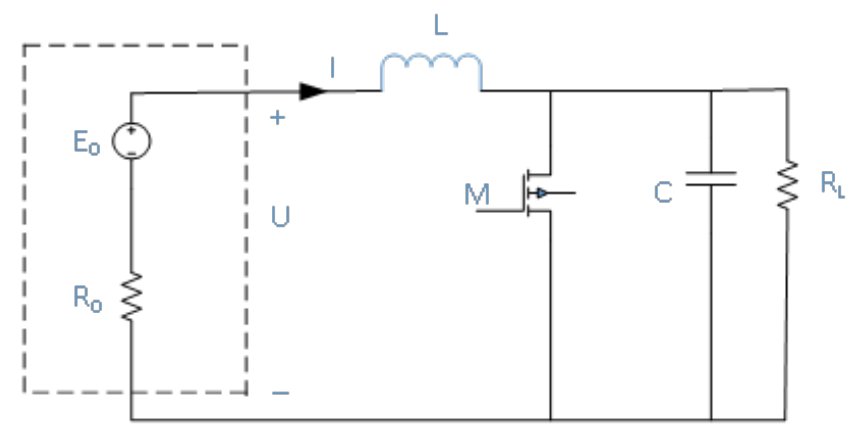

Fig. 6. Boost Circuit of Thermoelectric Generation

As shown in the Fig.6, when MOSFET is on, the voltage $E_{0}$ is:

$$
E_{0}=R_{0} I+L \frac{d I}{d t}
$$

Divided on both sides of the equation by $R_{0}$ :

$$
I_{s}=I+\frac{L}{R_{0}} \frac{d I}{d t}
$$

When the circuit works in MPPT,

$$
U_{s c}=\frac{E_{0}}{2}=I_{s} \frac{R_{0}}{2}
$$

Because $E_{0}$ will changes by different temperature, the short-circuit current of thermoelectric generator can be calculated according to the inductance current value and its changing rate. Then the value of $E_{0}$ can be calculated. So as long as the value of control port voltage $\mathrm{U}$ is $E_{0} / 2$, MPPT control strategy can be achieved.

$$
K=\frac{\left|U-U_{s c}\right|}{U_{s c}}
$$

When the working point is on the left of the maximum power point. 


$$
\frac{d P}{d U}>0: U=U+K \Delta U
$$

When the working point is the maximum power point.

$$
\frac{d P}{d U}=0: U=U_{m}
$$

When the working point is on the right of the maximum power point.

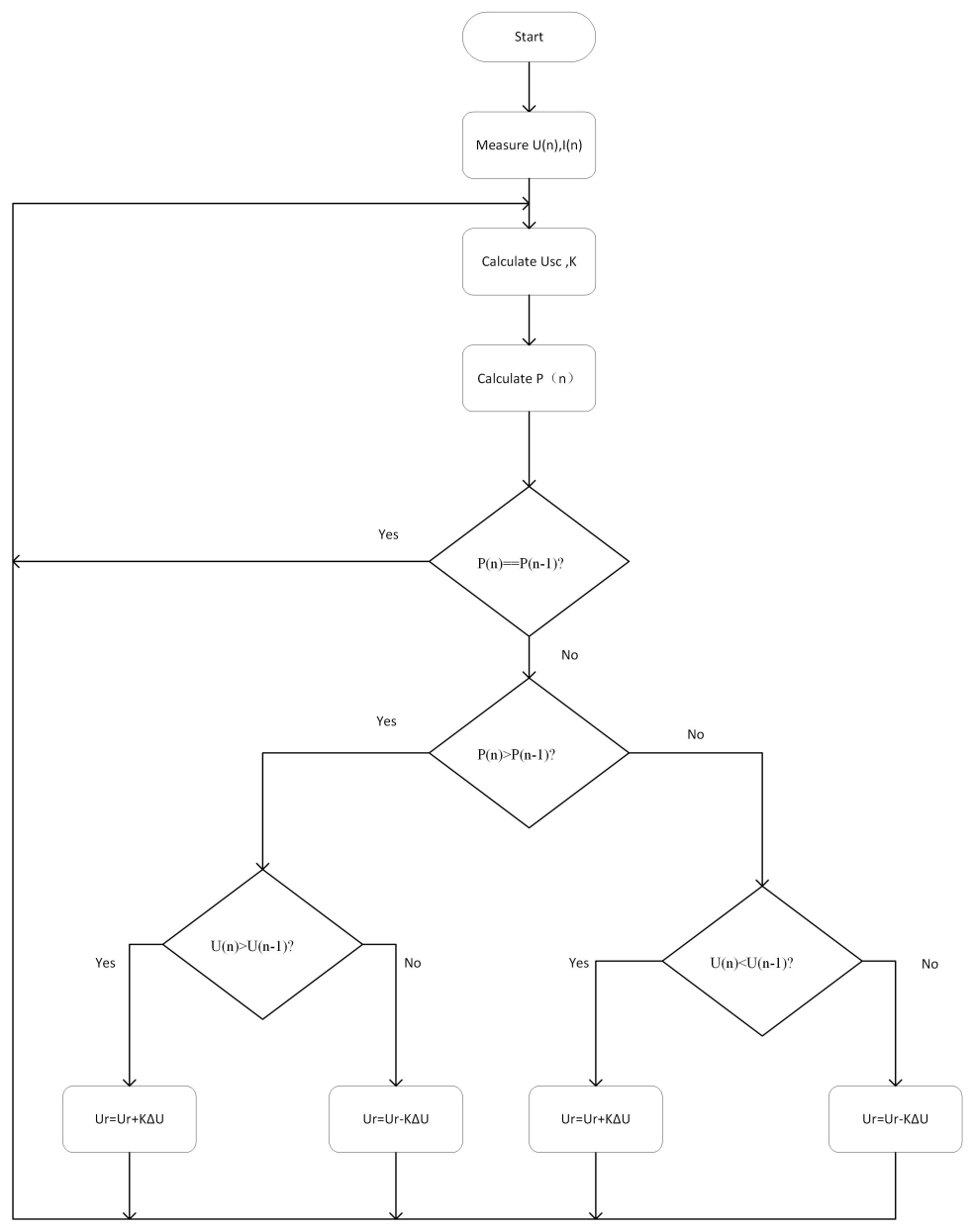

Fig. 7. Proportional Step Perturbation MPPT Flow Chart

$$
\frac{d P}{d U}<0: U=U-K \Delta U
$$


The Fig.7 is the proportional step perturbation MPPT flow chart. As shown in the Fig.7, the coefficient $\mathrm{K}$ corrects the new value in each cycle. Therefore, it can be ensured that the step size is larger when the working point is farther away from the MPPT point. And the step size is smaller when the working point is closer to the MPPT point.

\section{Simulation and conclusion}

The Fig. 8 is the I/U Waveform of the thermoelectric cell. And the Fig. 9 is the $\mathrm{P} / \mathrm{U}$ Waveform of the thermoelectric cell.

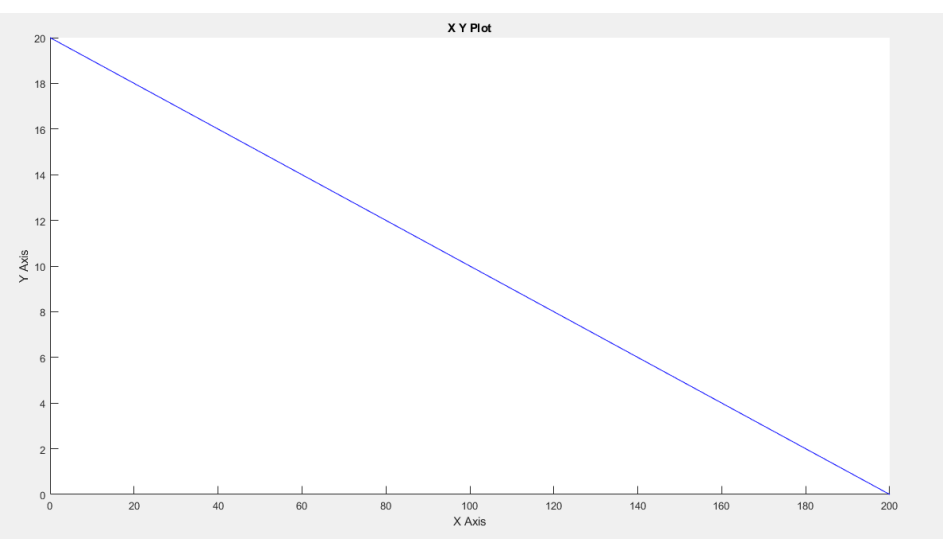

Fig. 8. I/U Waveform

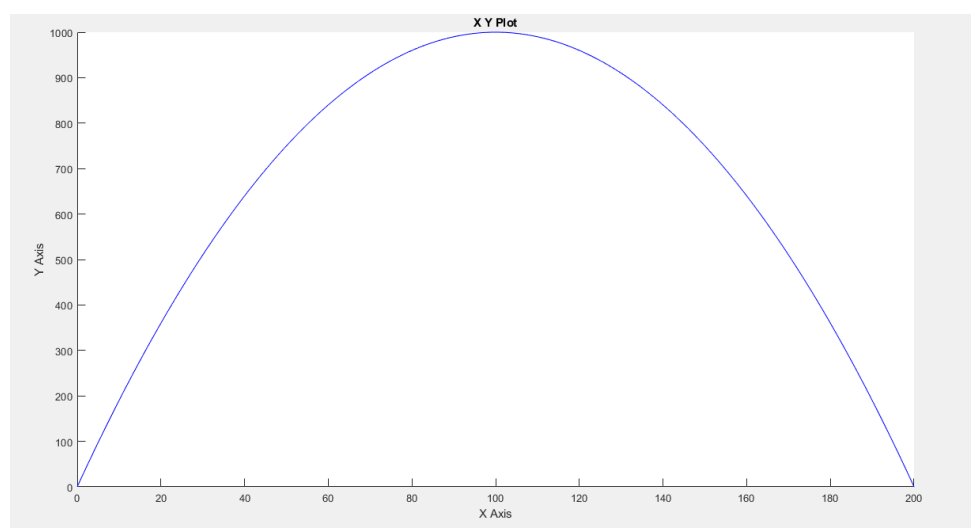

Fig. 9. P/U Waveform 
As shown in the Fig.9, the maximum power point is located near the midpoint of open circuit voltage. Therefore, the closer the voltage is to the midpoint, the smaller voltage step is to achieve accurate control. The further away from the midpoint of the open-circuit voltage, the voltage step can be increased for the purpose of quickly MPPT.

The Fig.10 is the simulation waveform of MPPT. The temperature changes at 0.03 seconds. And the maximum power changes from $1,400 \mathrm{~W}$ to $3,250 \mathrm{~W}$.

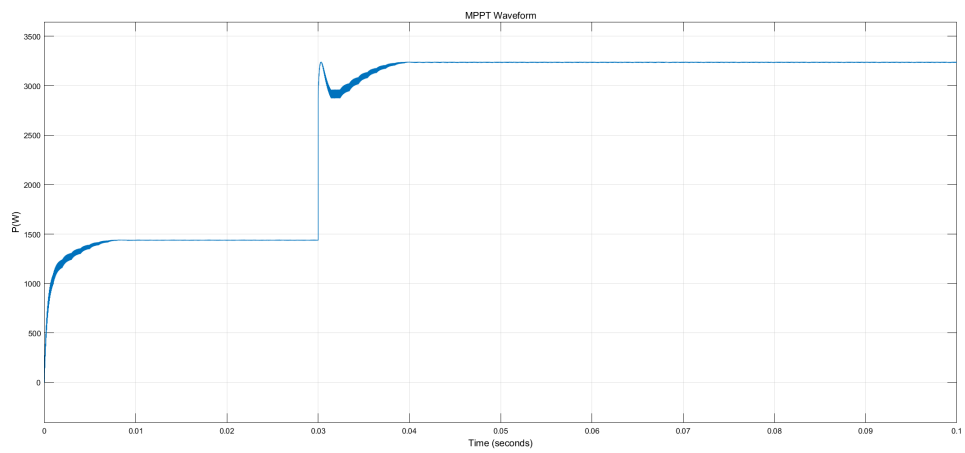

Fig. 10. MPPT Waveform

It can be seen from the waveform that the MPPT algorithm based on proportional step perturbation method can lock the maximum power point quickly, accurately and stably. The simulation results show that the design is feasible.

\section{References}

1. Y. Bai, B. Kou and C. C. Chan, "A Simple Structure Passive MPPT Standalone Wind Turbine Generator System," in IEEE Transactions on Magnetics, vol. 51, no. 11, pp. 1-4, Nov. 2015, Art no. 8204704.

2. S. Stanzione, C. van Liempd, R. van Schaijk, Y. Naito, F. Yazicioglu and C. Van Hoof, "A High Voltage Self-Biased Integrated DC-DC Buck Converter With Fully Analog MPPT Algorithm for Electrostatic Energy Harvesters," in IEEE Journal of Solid-State Circuits, vol. 48, no. 12, pp. 3002-3010, Dec. 2013.

3. S. Murdoch and S. Reynoso, "Design and Implementation of a MPPT circuit for a Solar UAV," in IEEE Latin America Transactions, vol. 11, no. 1, pp. 108-111, Feb. 2013.

4. R. Pradhan and B. Subudhi, "Double Integral Sliding Mode MPPT Control of a Photovoltaic System," in IEEE Transactions on Control Systems Technology, vol. 24, no. 1, pp. 285-292, Jan. 2016.

5. R. Jiang, Y. Han and S. Zhang, "Wide-range, high-precision and low-complexity MPPT circuit based on perturb and observe algorithm," in Electronics Letters, vol. 53, no. 16, pp. 1141-1142, 382017. 
6. M. Bond and J. Park, "Current-Sensorless Power Estimation and MPPT Implementation for Thermoelectric Generators," in IEEE Transactions on Industrial Electronics, vol. 62, no. 9, pp. 5539-5548, Sept. 2015.

7. O. Lopez-Santos et al., "Analysis, Design, and Implementation of a Static Conductance-Based MPPT Method," in IEEE Transactions on Power Electronics, vol. 34, no. 2, pp. 1960-1979, Feb. 2019.

8. X. Liu, L. Huang, K. Ravichandran and E. Snchez-Sinencio, "A Highly Efficient Reconfigurable Charge Pump Energy Harvester With Wide Harvesting Range and Two-Dimensional MPPT for Internet of Things," in IEEE Journal of Solid-State Circuits, vol. 51, no. 5, pp. 1302-1312, May 2016.

9. M. A. Abdullah, T. Al-Hadhrami, C. W. Tan and A. H. Yatim, "Towards Green Energy for Smart Cities: Particle Swarm Optimization Based MPPT Approach," in IEEE Access, vol. 6, pp. 58427-58438, 2018. 